\title{
Open
}

\section{Risks of breast or ovarian cancer in BRCA1 or BRCA2 predictive test negatives: findings from the EMBRACE study}

\author{
Fabio Girardi, $\mathrm{MD}^{1}$, Daniel R. Barnes, $\mathrm{PhD}^{1}$, Daniel Barrowdale, $\mathrm{BSC}^{1}$, Debra Frost, ONC ${ }^{1}$, \\ Angela F. Brady, FRCP PhD², Claire Miller, RN BA ${ }^{3}$, Alex Henderson, PhD ${ }^{4}$, Alan Donaldson, MD $^{5}$, \\ Alex Murray, $\mathrm{FRCP}^{6}$, Carole Brewer, $\mathrm{MD}^{7}$, Caroline Pottinger, MD ${ }^{8}$, D. Gareth Evans, MD ${ }^{9}$, \\ Diana Eccles, MD ${ }^{10}, \mathrm{EMBRACE}^{1}$, Fiona Lalloo, MD ${ }^{11}$, Helen Gregory, MD ${ }^{12}$, Jackie Cook, MD ${ }^{13}$, \\ Jacqueline Eason, $\mathrm{MD}^{14}$, Julian Adlard, MD PhD ${ }^{15}$, Julian Barwell, $\mathrm{MD}^{16}$, Kai Ren Ong, $\mathrm{MD}^{17}$, \\ Lisa Walker, PhD ${ }^{18}$, Louise Izatt, PhD ${ }^{19}$, Lucy E. Side, MD ${ }^{20}$, M. John Kennedy, MB ${ }^{21}$, \\ Marc Tischkowitz, MD PhD ${ }^{22}$, Mark T. Rogers, MD ${ }^{23}$, Mary E. Porteous, MD ${ }^{24}$, \\ Patrick J. Morrison, $\mathrm{MD}^{25}$, Ros Eeles, MD PhD ${ }^{26}$, Rosemarie Davidson, MD ${ }^{27}$, Katie Snape, MD ${ }^{28}$, \\ Douglas F. Easton, $\mathrm{PhD}^{1,29}$ and Antonis C. Antoniou, $\mathrm{PhD}^{1}$
}

Purpose: $B R C A 1 / B R C A 2$ predictive test negatives are proven noncarriers of a $B R C A 1 / B R C A 2$ mutation that is carried by their relatives. The risk of developing breast cancer (BC) or epithelial ovarian cancer (EOC) in these women is uncertain. The study aimed to estimate risks of invasive $\mathrm{BC}$ and $\mathrm{EOC}$ in a large cohort of $B R C A 1 / B R C A 2$ predictive test negatives.

Methods: We used cohort analysis to estimate incidences, cumulative risks, and standardized incidence ratios (SIRs).

Results: A total of 1,895 unaffected women were eligible for inclusion in the $\mathrm{BC}$ risk analysis and 1,736 in the EOC risk analysis. There were 23 incident invasive BCs and 2 EOCs. The cumulative risk of invasive BC was $9.4 \%$ (95\% confidence interval (CI) 5.9-15\%) by age 85 years and the corresponding risk of EOC was $0.6 \%$ (95\% CI $0.2-2.6 \%)$. The SIR for invasive BC was 0.93 (95\% CI $0.62-1.40$ ) in the overall cohort, 0.85 (95\% CI $0.48-1.50)$ in noncarriers from BRCA1 families, and 1.03 (95\% CI 0.57-1.87) in noncarriers from BRCA2 families. The SIR for EOC was 0.79 (95\% CI 0.20-3.17) in the overall cohort.

Conclusion: Our results did not provide evidence for elevated risks of invasive $\mathrm{BC}$ or EOC in BRCA1/BRCA2 predictive test negatives.

Genet Med advance online publication 22 March 2018

Key Words: BRCA1/BRCA2; breast cancer; ovarian cancer; predictive test negatives; risks

\section{INTRODUCTION}

Several breast and ovarian cancer susceptibility genes have been identified to date. The most important genes in the context of genetic counseling are BRCA1 and BRCA2, which are associated with high risks of developing breast, ovarian, and other cancers. ${ }^{1-5}$ Genetic testing for BRCA1 and BRCA2 has become an integral part of genetic counseling; the results are used to inform women's treatment or clinical management options, which involve a combination of screening, prophylactic surgery, and other risk reduction strategies. $^{6-11}$

$B R C A 1 / B R C A 2$ predictive test negatives are individuals who have been offered a predictive test for a specific BRCA mutation that has been found in a biological relative, and then found to be a noncarrier. Several studies have estimated the risk of developing breast cancer (BC) and epithelial ovarian

\footnotetext{
${ }^{1}$ Centre for Cancer Genetic Epidemiology, Department of Public Health and Primary Care, University of Cambridge, Cambridge, UK; ${ }^{2}$ North West Thames Regional Genetics Service, Northwick Park Hospital, London North West Healthcare NHS Trust, Harrow, UK; ${ }^{3}$ Cheshire and Merseyside Clinical Genetics Service, Liverpool Women's NHS Foundation Trust, Liverpool, UK; ${ }^{4}$ Institute of Genetic Medicine, Centre for Life, Newcastle Upon Tyne Hospitals NHS Trust, Newcastle upon Tyne, UK; ${ }^{5}$ Clinical Genetics Department, St Michael's Hospital, Bristol, UK; ${ }^{6}$ All Wales Medical Genetics Services, Singleton Hospital, Swansea, UK; ${ }^{7}$ Department of Clinical Genetics, Royal Devon \& Exeter Hospital, Exeter, UK; ${ }^{8}$ All Wales Medical Genetics Service, Glan Clwyd Hospital, Rhyl, UK; ${ }^{9}$ Manchester Centre for Genomic Medicine, Manchester Academic Health Sciences Centre, Division of Evolution and Genomic Science, Manchester University, Manchester Universities NHS Foundation Trust, Manchester, UK; ${ }^{10}$ University of Southampton Faculty of Medicine, Southampton University Hospitals NHS Trust, Southampton, UK; ${ }^{11}$ Manchester Centre for Genomic Medicine, Manchester Academic Health Sciences Centre, Manchester Universities NHS Foundation Trust, Manchester, UK; ${ }^{12}$ North of Scotland Regional Genetics Service, NHS Grampian \& University of Aberdeen, Foresterhill, Aberdeen, UK; ${ }^{13}$ Sheffield Clinical Genetics Service, Sheffield Children's Hospital, Sheffield, UK; ${ }^{14}$ Nottingham Clinical Genetics Service, Nottingham University Hospitals NHS Trust, Nottingham, UK; ${ }^{15}$ Yorkshire Regional Genetics Service, Chapel Allerton Hospital, Leeds, UK; ${ }^{16}$ Leicestershire Clinical Genetics Service, University Hospitals of Leicester NHS Trust, Leicester, UK; ${ }^{17}$ West Midlands Regional Genetics Service, Birmingham Women's Hospital Healthcare NHS Trust, Birmingham, UK; ${ }^{18}$ Oxford Regional Genetics Service, Churchill Hospital, Oxford, UK; ${ }^{19}$ Clinical Genetics, Guy's and St. Thomas' NHS Foundation Trust, London, UK; ${ }^{20}$ North East Thames Regional Genetics Service, Great Ormond Street Hospital for Children NHS Trust, London, UK; ${ }^{21}$ Academic Unit of Clinical and Molecular Oncology, Trinity College Dublin and St James's Hospital, Dublin, Ireland; ${ }^{22}$ Department of Medical Genetics, University of Cambridge, Cambridge, UK; ${ }^{23}$ All Wales Medical Genetics Services, University Hospital of Wales, Cardiff, UK; ${ }^{24}$ South East of Scotland Regional Genetics Service, Western General Hospital, Edinburgh, UK; ${ }^{25}$ Centre for Cancer Research and Cell Biology, Queens University of Belfast, Belfast, UK;

${ }^{26}$ Oncogenetics Team, The Institute of Cancer Research and Royal Marsden NHS Foundation Trust, London, UK; ${ }^{27}$ Department of Clinical Genetics, South Glasgow University Hospitals, Glasgow, UK; ${ }^{28}$ Medical Genetics Unit, St George's, University of London, London, UK; ${ }^{29}$ Centre for Cancer Genetic Epidemiology, Department of Oncology, University of Cambridge, Cambridge, UK. Correspondence: Antonis C. Antoniou (aca20@medschl.cam.ac.uk)
} 
cancer (EOC) in these women, but estimates vary widely across studies. Although the risks have generally been found to be lower than those in BRCA1 and BRCA2 mutation carriers, retrospective studies have estimated the risks of $\mathrm{BC}$ to be two- to fivefold higher than the risk in the general population. ${ }^{12,13}$ Estimates based on retrospective studies may be overestimated if ascertainment is not correctly allowed for in the analysis. The findings from the eight prospective studies published to date have been conflicting. In five of them, risk to noncarriers was not raised (0.52-0.95) (refs. 1418) while in three studies the estimated risks were significantly increased (2.0-4.57) (refs. 19-21). An important limitation in all these studies, however, was small sample size, and as a result the point estimates have wide confidence intervals. Data on EOC risks for BRCA1/BRCA2 predictive test negatives are currently scarce and the only estimates available are from retrospective studies. ${ }^{13,22}$

Here, we used data from a large prospective cohort of $B R C A 1 / B R C A 2$ predictive test negatives from the United Kingdom to estimate the risks of developing BC or EOC.

\section{Subjects}

\section{MATERIALS AND METHODS}

The Epidemiological Study of Familial Breast Cancer (EMBRACE) study is a prospective cohort study in the United Kingdom and Republic of Ireland, aiming to characterize cancer risks in $B R C A 1 / B R C A 2$ mutation carriers and their relatives. Individuals are eligible for inclusion in EMBRACE if they (i) carry a pathogenic mutation in BRCA1 or $B R C A 2$, (ii) are noncarriers in families with a pathogenic $B R C A 1$ or $B R C A 2$ mutation, or (iii) are potential carriers but opt not to undergo a predictive genetic test. Recruitment is organized through regional cancer genetics centers, generally close to the time of genetic testing. Recruitment commenced in 1997 and includes both women and men. The cohort includes individuals with or without personal history of cancer; all participants complete a baseline questionnaire that includes information on $\mathrm{BC}$ and $\mathrm{EOC}$ risk factors and medical history. The EMBRACE study was approved by the former Anglia and Oxford Medical Research and Ethics Committee (North West Anglia Health Authority, Peterborough, United Kingdom), now East of England-Cambridge South Research Ethics Committee (Nottingham, United Kingdom).

Only noncarrier women were eligible for inclusion in the present study. Information on date and cause of death, and cancer diagnoses, was obtained through linkage with the Health and Social Care Information Centre for England and Wales, and with the National Health Service Central Register for Scotland. For the purpose of the study, the last record linkage was performed on 15 December 2015. The end of follow-up was set as 15 July 2015 to ensure that any cancer diagnoses by this date were likely to have been reported at the time of the record linkage.

Cancer risks for $B R C A 1 / B R C A 2$ predictive test negatives were estimated separately for incident invasive $B C$ and incident EOC. Women were eligible for inclusion in the analysis if they had not been diagnosed with cancer, other than nonmelanoma skin cancer, before the date at the baseline questionnaire (study recruitment). For the estimation of $\mathrm{BC}$ risk, participants were ineligible if they had already undergone risk-reducing bilateral mastectomy at recruitment. In estimating EOC risk, participants were ineligible if they had undergone risk-reducing salpingo-oophorectomy (RRSO) or bilateral salpingectomy (because noncarriers in EMBRACE do not complete follow-up questionnaires after study recruitment, information on prospective prophylactic surgeries occurring after the baseline questionnaire date was not available). Women were followed from baseline until the first of (i) death, (ii) a diagnosis of a cancer other than nonmelanoma skin cancer, (iii) attained age 85 years, or (iv) 15 July 2015. For the BC risk estimation, only women diagnosed with an incident invasive $\mathrm{BC}$ were assumed to be affected (i.e., noninvasive $\mathrm{BCs}$ were ignored but censored as unaffected at diagnosis) and for the EOC analysis only women diagnosed with an EOC were assumed to be affected.

\section{Statistical analysis}

Annual incidences of invasive $\mathrm{BC}$ and EOC per 1,000 personyears were estimated for the age intervals 18-24, 25-34, 35$44,45-54,55-64,65-74$, and 75-84 using standard cohort analysis. Kaplan-Meier analysis was used to estimate the cumulative risks. The probability of experiencing the event of interest up to a given time-point was calculated as 1-KaplanMeier estimate of the survival probability. Differences in cancer risks between members of BRCA1 families and members of BRCA2 families were assessed using Cox regression. The hazard ratio was defined as the ratio between the hazard function at age $t$ for noncarriers from $B R C A 2$ families and the hazard function at age $t$ for noncarriers from $B R C A 1$ families. To account for possible variation in risk due to year of birth (i.e., cohort effect), the analysis was adjusted for year of birth using a categorical variable based on the observed quartiles (1922 to 1953, 1954 to 1963,1964 to 1972, 1973 to 1995).

We assessed the difference between the estimated incidence rates of invasive $\mathrm{BC}$ and $\mathrm{EOC}$ in the sample under study and the corresponding incidence rates in a reference population by estimating the standardized incidence ratio (SIR). A SIR is the ratio between the number of observed incident cases and the number of expected incident cases in the study population over the period of observation. For this purpose, we used the population incidences for England ${ }^{23}$ and assumed they also apply to women recruited from Scotland and Wales. Expected cases were calculated by applying the calendar periodspecific, age-specific, and cancer-specific incidences for England to the eligible sample of noncarriers. These rates were available from 1998 to 2014. Rates for 1998 and 2014 were assumed to apply for the years 1997 and 2015, respectively. To allow for the fact that multiple individuals from the same family may be included in the analyses robust standard errors were calculated by clustering on family. ${ }^{24}$ 


\section{RESULTS}

The process of generating the eligible sets for inclusion in the $\mathrm{BC}$ and EOC analyses are described in the appendix (Supplementary Figures S1 and S2 online). Table 1 summarizes the eligible cohort characteristics.

\section{Risk of invasive BC}

Among 1,895 BRCA1/BRCA2 predictive test negatives eligible for inclusion in the $\mathrm{BC}$ analysis, 23 incident invasive $\mathrm{BCs}$ occurred, 12 in women from BRCA1 families and 11 in women from BRCA2 families. The estimated incidences, cumulative risks, and SIRs are shown in Table 2. The crude incidence rate of invasive $\mathrm{BC}$ in the overall sample was estimated to be 1.72 per 1,000 person-years (95\% confidence interval (CI) 1.16-2.66). The incidence rate increased rapidly at young ages up to age 45 but remained relatively constant after that age, ranging from 2.33 to 3.04 per 1,000 person-years.
The estimate was similar when analysis was restricted to individuals of European ancestry (1.74/1,000 person-years, 95\% CI 1.17-2.70). The incidence rate in members of BRCA2 families $(1.95 / 1,000$ person-years, 95\% CI 1.11-3.79) was slightly, but not significantly ( $p$ value 0.58 ), higher than the rate in members of BRCA1 families (1.55/1,000 person-years, 95\% CI 0.90-2.92). After adjustment for birth cohort, the hazard ratio estimate for members of $B R C A 2$ families versus members of BRCA1 families was 1.20 (95\% CI $0.53-2.70$, Wald test $p$ value $=0.6$ ). The SIR estimate for invasive $B C$ was 0.93 (95\% CI 0.62-1.40). In the age-specific analysis the highest SIR was estimated for age group $45-55$ (SIR $=1.30$, 95\% CI 0.72-2.35), but SIRs were estimated to be $<1$ for all other age groups (range: 0.35-0.89). There was no apparent trend in the SIRs with age. The SIR was estimated to be 0.85 (95\% CI 0.48-1.50) for members of a BRCA1 family with the highest SIR estimated for age group 65-75 ( $\mathrm{SIR}=1.56,95 \%$

Table 1 Descriptive summary of the cohorts in which risks were calculated, separately for invasive BC and EOC

\begin{tabular}{|c|c|c|c|c|c|c|}
\hline & \multicolumn{3}{|c|}{ Eligible set for invasive $B C$ analysis } & \multicolumn{3}{|c|}{ Eligible set for EOC analysis } \\
\hline Mean age at questionnaire (SD) & $45.1(13.1)$ & $44.6(13.1)$ & $45.6(13)$ & $44.2(13.1)$ & $43.5(13)$ & $45.1(13.1)$ \\
\hline Failures & 23 & 12 & 11 & 2 & 0 & 2 \\
\hline Mean age at failure (SD) & $57.2(8.8)$ & $60.5(9.7)$ & $53.6(6.2)$ & $50.5(8.0)$ & - & $50.5(8.0)$ \\
\hline White & $1,810(96)$ & $982(94)$ & $824(97)$ & $1,658(95)$ & $885(94)$ & $769(97)$ \\
\hline Indian & $5(0.3)$ & $4(0.4)$ & $1(0.1)$ & $5(0.4)$ & $4(0.5)$ & $1(0.1)$ \\
\hline Black-Caribbean & $3(0.2)$ & $3(0.3)$ & 0 & $3(0.2)$ & $3(0.3)$ & 0 \\
\hline Ashkenazi Jewish & $47(2)$ & $34(3.4)$ & $13(1.8)$ & $45(3)$ & $32(3.4)$ & $13(1.8)$ \\
\hline Bangladeshi & $1(0)$ & $1(0.1)$ & 0 & $1(0)$ & $1(0.1)$ & 0 \\
\hline Other & $19(1)$ & $11(1.1)$ & $8(0.9)$ & $16(0.9)$ & $8(1)$ & $8(1)$ \\
\hline Number of families (\%) & 1,602 & $887(55)$ & $711(45)$ & 1,488 & $812(55)$ & $672(45)$ \\
\hline \multicolumn{7}{|c|}{ Families by number of noncarriers (\%) } \\
\hline 1 & $1,381(86)$ & $767(86)$ & $610(86)$ & $1,300(87)$ & $714(88)$ & $582(88)$ \\
\hline 2 & $179(11)$ & $98(11)$ & $81(11)$ & $152(11)$ & $80(10)$ & $72(11)$ \\
\hline 3 & $25(2)$ & $14(2)$ & $11(1.6)$ & $24(1.5)$ & $11(1.3)$ & $13(0.3)$ \\
\hline 4 & $14(1)$ & $6(0.8)$ & $8(1.3)$ & $9(0.5)$ & $5(0.5)$ & $4(0.6)$ \\
\hline 5 & $1(0)$ & $1(0.1)$ & 0 & $1(0)$ & $1(0.1)$ & 0 \\
\hline 6 & 0 & 0 & 0 & 0 & 0 & 0 \\
\hline 7 & 0 & 0 & 0 & $1(0)$ & $1(0.1)$ & 0 \\
\hline
\end{tabular}

BC, breast cancer; EOC, epithelial ovarian cancer. 'In four individuals family identification number and family mutation were missing. In reference to familial clustering, these individuals were assumed to be independent. No assumptions were made on family mutation; therefore, in the stratified analysis by family mutation, these individuals were not considered. 


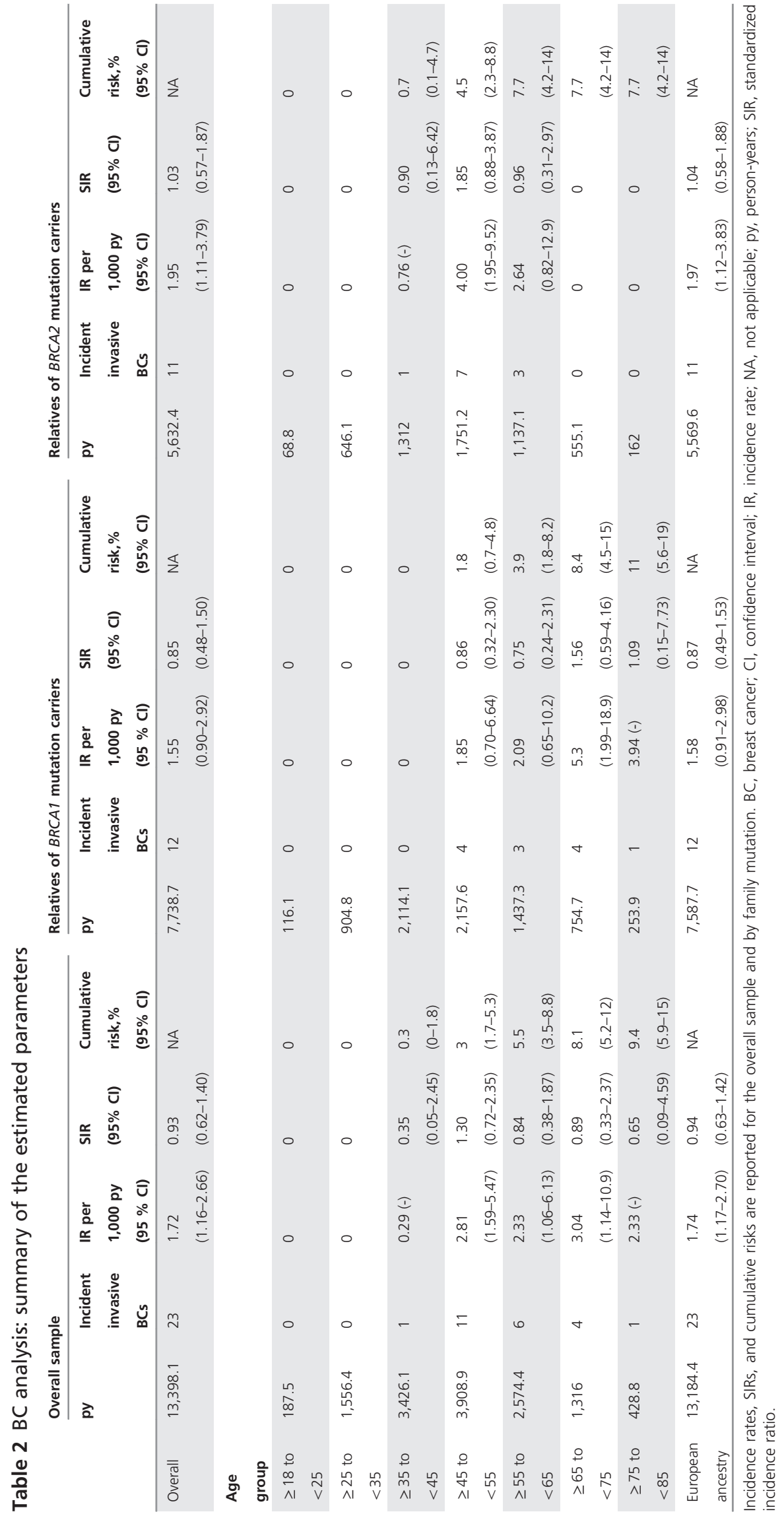




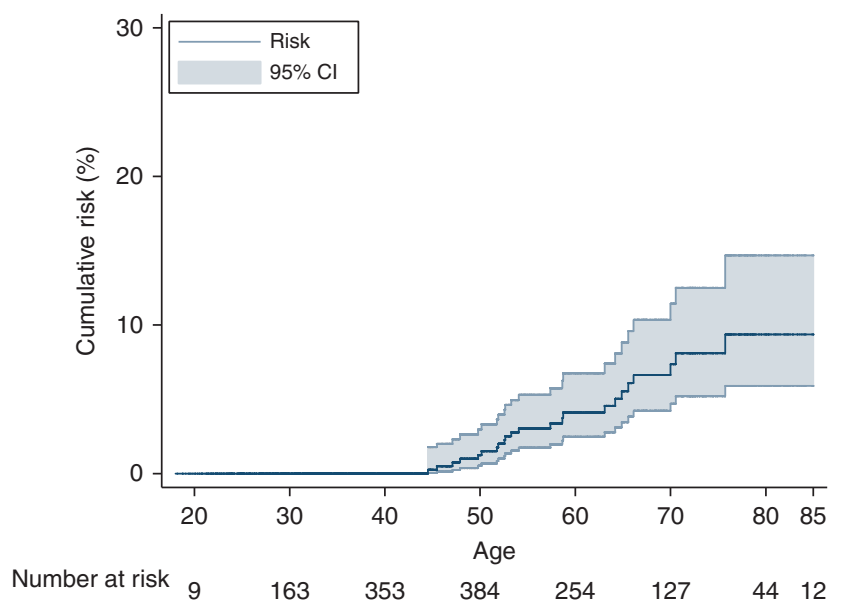

Figure 1 Kaplan-Meier plot for the risk of invasive breast cancer in the combined sample of noncarriers from families with BRCA1 and BRCA2 mutations. $\mathrm{Cl}$, confidence interval.

CI 0.59-4.16) and 1.03 (95\% CI 0.57-1.87) for relatives of BRCA2 mutation carriers with the highest SIR occurring for ages $45-55$ (SIR $=1.85,95 \%$ CI 0.88-3.87) (Table 2). The risk of developing invasive $\mathrm{BC}$ in the pooled sample was estimated to be $3 \%$ (95\% CI $1.7-5.3 \%)$ by age 55 years and $9.4 \%$ (95\% CI $5.9-15 \%$ ) by age 85 years (Figure 1). When analysis was restricted to members of BRCA1 families, the cumulative risk of invasive $\mathrm{BC}$ was estimated to be $1.8 \%$ (95\% CI $0.7-$ $4.8 \%$ ) by age 55 and $11 \%$ (95\% CI $5.6-19 \%)$ by age 85 . For members of BRCA2 families, the risk of developing invasive BC was $4.5 \%$ (95\% CI 2.3-8.8\%) by age 55 and $7.7 \%$ (95\% CI $4.2-14 \%$ ) by age 85 (Supplementary Figure S3 online). Sensitivity analyses, after excluding women with bilateral oophorectomy, excluding the first year of follow-up or by including in situ BCs yielded similar results (sensitivity analyses described in the appendix (Supplementary Table S1 online)).

\section{Risk of EOC}

In the cohort of 1,736 noncarriers eligible for the EOC analysis, two incident EOCs were observed, both in individuals from $B R C A 2$ families, with ages at diagnosis 44.8 and 56.1 years. The crude EOC incidence rate in the overall sample was 0.16 per 1,000 person-years (95\% CI $0.03-$ 1.66). The estimated SIR for EOC was 0.79 (95\% CI $0.20-$ 3.17) for all age groups. With only two incident EOCs, SIR estimates by age group and by family mutation were either not possible or associated with wide confidence intervals. They are not therefore reported. The estimated absolute risk of EOC in noncarriers was $0.6 \%$ (95\% CI $0.2-2.6 \%$ ) by age 85 years. In a sensitivity analysis that included women with a prior BC diagnosis in the eligible cohort, results were unchanged (Supplementary Material online).

\section{DISCUSSION}

This is the largest prospective cohort study of BRCA1/BRCA2 predictive test negatives to date. We found no evidence of an increased $\mathrm{BC}$ or $\mathrm{EOC}$ risk in relatives of $B R C A 1$ or $B R C A 2$ mutation carriers. The $B C$ risk estimates were not influenced by the inclusion of individuals with prior bilateral salpingooophorectomy. When the four noninvasive prospective BCs were also considered as events, to rule out the possibility that some cancers were being wrongly excluded, the SIR estimates were similar. Similarly, after censoring individuals at an earlier date (15 June 2014) to allow for possible risk underestimation due to delayed cancer notification, results were unchanged.

Early clinical recommendations on hereditary BC and EOC recommended standard surveillance for BRCA1/BRCA2 predictive test negatives. ${ }^{25}$ In 2013, the United States Preventive Services Task Force issued a recommendation reiterating that noncarriers were at population risk. ${ }^{26,27}$ According to the National Institute for Health and Care Excellence guidelines, ${ }^{6}$ women at population risk should be given standard surveillance and discussion of any further riskreducing intervention would not be appropriate. If, however, $B R C A 1 / B R C A 2$ predictive test negatives are at increased risk of developing $\mathrm{BC}$, as recently suggested, ${ }^{19-21}$ the recommendations for women at moderate to high risk of developing $\mathrm{BC}$ may apply.

In five of the eight prospective studies published to date, the estimated SIR for BC was in line with our results (range of SIRs $=0.52-0.95)$ (refs. 14-18). In three studies a significant two- to fivefold increased risk of BC was reported compared with population risks, but there were inconsistencies between these studies. ${ }^{19-21}$ Evans et al. ${ }^{19}$ found a statistically significant increased risk only for relatives of BRCA2 mutation carriers, while Vos et al. ${ }^{21}$ found a statistically significant increased risk only for relatives of BRCA1 mutation carriers. Both studies only included first-degree relatives of known mutation carriers. The authors argued that common genetic variants, which modify risks in BRCA1/BRCA2 carriers, also may modify risk in noncarriers. These predisposing variants would be more likely to segregate in families with multiple affected individuals, such as those ascertained in genetics clinics. Korde et al. ${ }^{17}$ and Nielsen et al., ${ }^{18}$ however, did not find an increase in the risk of $\mathrm{BC}$ for $B R C A$ predictive test negatives who were first-degree relatives of mutation carriers. A limitation of all these studies was the small sample size (none included more than $21 \mathrm{BC}$ cases). In the present study we were not able to restrict analysis to only first-degree relatives of known mutation carriers because the exact familial relationships were not available. Similarly, we were not able to investigate the variation in risk by family history of cancer. Korde et al. ${ }^{17}$ reported a nonsignificant increase in the risk of $\mathrm{BC}$ for noncarriers with an affected first-degree relative. In Harvey et al. ${ }^{16}$ the estimated SIR of $\mathrm{BC}$ in predictive test negatives without affected relatives in the parental lineage not associated with the $B R C A$ mutation was 0.48 , albeit statistically not significant.

Only a few published studies provided a SIR for EOC (range of SIRs reported: 0-4.6) (refs. 13,22) but all were based on retrospective studies. Three prospective studies ${ }^{15,20,21}$ aimed 
to estimate a SIR for EOC. In Domchek et al. ${ }^{15}$ and Rowan et al. ${ }^{20}$ no events occurred during the follow-up, while Vos et al. ${ }^{21}$ reported two EOCs but the corresponding SIR was not provided. This is the first study to estimate a SIR for EOC based on a prospective cohort, but the confidence intervals were wide due to the small number of events.

The sample size of this study is larger than previous studies and the confidence limits are correspondingly narrower. Nonetheless, when considering $\mathrm{BC}$ risk estimates by age group and family mutation, caution is needed due to the relatively small number of events in each stratum. Because this is a prospective cohort, estimates are less likely to be prone to ascertainment or reporting biases, usually associated with retrospective or historical cohort studies.

Limitations of this study include the following:

1. The SIRs were calculated using incidences from England, but EMBRACE noncarriers were also recruited in Scotland and Wales. Age-specific estimates for these countries were not readily available; however, data from previous periods indicate that the incidence rates are similar amongst countries. Moreover, the large majority of study participants (83.3\%) were from clinics based in England.

2. Surgeries unrelated to cancer diagnoses prior to recruitment were not verified through surgical records, but bias due to misreporting or underreporting was probably small given the life-changing sexual and physical impact of risk-reducing surgery. ${ }^{28-30}$

3. No information on prophylactic surgeries after recruitment was available. Such surgeries are unlikely in women testing negative for mutations; however, if risk-reducing surgeries are more frequent in the noncarriers within families with BRCA1 and BRCA2 mutations compared with the general population this may lead to an underestimation of the SIR. In an approximate calculation, assuming the same proportion (i.e., 0.88\%) of bilateral mastectomies in the prospective cohort as in the retrospective data, 17 such procedures would be expected. In the unlikely event that all these individuals would have developed invasive BC if they had not undergone surgery, the observed/expected ratio would have been 1.61 (95\% CI 1.18-2.2). In the retrospective data, $5.4 \%$ of noncarriers from BRCA1 families had undergone bilateral oophorectomy with or without bilateral salpingectomy, compared with $3.3 \%$ of noncarriers from $B R C A 2$ families, possibly reflecting differential counseling toward risk-reducing surgery when a $B R C A 1$ mutation segregates in the family. In the current sample, $98 \%$ of noncarriers from BRCA1 families who underwent bilateral oophorectomy (or bilateral salpingectomy) at baseline had the procedure before genetic testing, whereas only $3 \%$ underwent RRSO after notification of the negative result. If the uptake of RRSO (after notification of mutation carrier status) was similar in the prospective cohort, this may have led to some underestimation of the EOC SIR for relatives of BRCA1 mutation carriers and in the overall sample. However, the size of these biases is most likely small because riskreducing bilateral mastectomy and RRSO after the disclosure of a negative result are less likely.

4. The noncarrier's degree of relationship with the mutation carrier and cancer family history were not available. Although women were ascertained through clinical genetics centers and most would have come from highrisk families, ascertainment criteria with respect to family history can vary. Therefore, it is not possible to rule out that risk in noncarriers may be increased if multiple family members or first-degree relatives are affected.

5. No information on the date of the most recent screening mammogram or breast magnetic resonance imaging was available. If $B R C A 1 / B R C A 2$ predictive test negatives with a normal breast imaging close to the genetic test discontinue surveillance following the notification of noncarrier status, they may be subject to a reverse lead time effect if they are diagnosed with a $\mathrm{BC}$ and this may lead to some underestimation of the risk. We investigated this by calculating the $\mathrm{BC}$ incidence in 3 -year intervals following the genetic test. Although there was a suggestion of a higher BC incidence rate between 3 and 6 years following the genetic test compared with the initial years, the differences were not significant. Larger sample sizes will be required to clarify this potential bias.

6. Although this is the largest prospective study, the mean follow-up time in the cohort was still relatively short (7.1 years) and longer follow-up will be required to investigate the risks at older ages with more precision.

Based on the confidence interval of the invasive BC SIRs, a 1.5 -fold increase in risk in noncarriers from $B R C A 1$ families and a 1.9-fold increase in noncarriers from $B R C A 2$ families cannot be ruled out. Translating these SIRs to lifetime risk yields an upper bound of risk of $\sim 17 \%$ to age 80 years for relatives of BRCA1 mutation carriers and $\sim 20 \%$ to age 80 years for relatives of $B R C A 2$ mutation carriers, based on UK general population incidences. ${ }^{31}$ Hence, even at the upper bound of the $95 \%$ CI, BRCA1/BRCA2 predictive test negatives would not be classified as being at high risk of developing invasive $\mathrm{BC}$ according to the National Institute for Health and Care Excellence guidelines. ${ }^{6}$

The familial aggregation of $\mathrm{BC}$ is known to be determined by other factors, including multiple common genetic variants, in addition to BRCA1 and BRCA2 mutations. Thus, one would predict that $B R C A 1 / B R C A 2$ predictive test negatives in cohorts such as the present study, who have a family history of $\mathrm{BC}$ and are hence more likely to carry other predisposing variants, would still be at increased risk relative to general population. We therefore investigated whether the present estimates are consistent with predictions given by the Breast and Ovarian Analysis of Disease Incidence and Carrier 
Estimation Algorithm (BOADICEA) model, which models the familial aggregation of $\mathrm{BC}$ in terms of $B R C A 1 / 2$ mutation status and a residual polygenic component. We computed the predicted lifetime risk of $\mathrm{BC}$ for a 20 -year-old true negative, with two different family histories: (i) a mutation-positive mother diagnosed with $\mathrm{BC}$ at age 50 years, and (ii) a mother and maternal grandmother both diagnosed with $\mathrm{BC}$ at age 50 and carrying a mutation. The predicted $\mathrm{BC}$ risks to age 80 years in these two scenarios were 12 and $13 \%$ for a BRCA1 positive family, and 14 and $15 \%$ for a $B R C A 2$ positive family. These women would be classified as at near population risk according to BOADICEA. Nevertheless, the predicted risks are somewhat higher than the point estimates in the present study, but within or close to the upper confidence interval bounds of the estimated cumulative risks. These observations suggest that the BOADICEA model may overestimate slightly the risks to individuals with a negative predictive test. However, larger sample sizes will be required to resolve this issue: if the true risks were in line with those predicted by BOADICEA, a prospective study with at least 100 events would be required to provide sufficient statistical power.

Our results suggest that $B R C A 1 / B R C A 2$ predictive test negatives can be classified as being at near-population risk as defined by the National Institute for Health and Care Excellence guidelines. ${ }^{6}$ Therefore, it would not be appropriate to offer risk-reducing bilateral mastectomy and RRSO to these individuals. In practice a proportion of women undergo preventative surgery based solely on the family history. Based on these data, predictive genetic testing of female relatives of a known BRCA1/BRCA2 mutation carrier is strongly beneficial in terms of avoiding unnecessary surgical procedures for those found to be BRCA1/BRCA2 negative. Standard surveillance may be offered, unless personal risk factors warrant further consideration such as strong family history of cancer in the parental lineage not associated with the known mutation or history of lobular carcinoma in situ or atypical ductal hyperplasia. ${ }^{32,33}$

\section{SUPPLEMENTARY MATERIAL}

Supplementary material is linked to the online version of the paper at http://www.nature.com/gim

\section{ACKNOWLEDGMENTS}

This work was supported by Cancer Research-UK grants C12292/ A20861, C1287/A10118, C1287/A11990, C1287/A23382, C1287/A17523, and C1287/A16563.

\section{DISCLOSURE}

D.E. has received honoraria from AstraZeneca and has reported consulting or advisory role in AstraZeneca. The other authors declare no conflict of interest.

\section{REFERENCES}

1. Miki Y, Swensen J, Shattuck-Eidens D, et al. A strong candidate for the breast and ovarian cancer susceptibility gene BRCA1. Science 1994;266:66-71.

2. Wooster R, Bignell $G$, Lancaster J, et al. Identification of the breast cancer susceptibility gene BRCA2. Nature 1995;378:789-792.
3. Antoniou AC, Cunningham AP, Peto J, et al. The BOADICEA model of genetic susceptibility to breast and ovarian cancers: updates and extensions. Br J Cancer 2008;98:1457-1466.

4. The Breast Cancer Linkage Consortium. Cancer risks in BRCA2 mutation carriers. J Natl Cancer Inst 1999;91:1310-1316.

5. Antoniou A, Pharoah PD, Narod S, et al. Average risks of breast and ovarian cancer associated with BRCA1 or BRCA2 mutations detected in case series unselected for family history: a combined analysis of 22 studies. Am J Hum Genet 2003;72:1117-1130.

6. The National Institute for Health and Care Excellence. Familial breast cancer: classification, care and managing breast cancer and related risks in people with a family history of breast cancer, 2013. https://www. nice. org.uk/Guidance/CG164. Accessed 1 March 2017.

7. National Comprehensive Cancer Network. NCCN guidelines for detection, prevention, \& risk reduction, 2016. https://www.nccn.org/ professionals/physician_gls/pdf/breast_risk.pdf. Accessed 1 March 2017.

8. Karlan BY, Berchuck, A \& Mutch D. The role of genetic testing for cancer susceptibility in gynecologic practice. Obstet Gynecol 2007;110: 155-167.

9. Greene MH, Mai PL \& Schwartz PE. Does bilateral salpingectomy with ovarian retention warrant consideration as a temporary bridge to risk-reducing bilateral oophorectomy in BRCA1/2 mutation carriers? Am J Obstet Gynecol 2011;204:19.e1-6.

10. Walker JL, Powell CB, Chen LM, et al. Society of Gynecologic Oncology recommendations for the prevention of ovarian cancer. Cancer 2015; 121:2108-2120.

11. Hartmann LC \& Lindor NM. The role of risk-reducing surgery in hereditary breast and ovarian cancer. N Engl J Med 2016;374:454-468.

12. Gronwald J, Cybulski C, Lubinski J \& Narod SA. Phenocopies in breast cancer 1 (BRCA1) families: implications for genetic counselling. J Med Genet. 2007;44:e76.

13. Smith A, Moran A, Boyd MC, et al. Phenocopies in BRCA1 and BRCA2 families: evidence for modifier genes and implications for screening. J Med Genet 2007:44:10-15.

14. Bernholtz S, Laitman $Y$, Kaufman B, Shimon-Paluch S \& Friedman E. Phenocopy breast cancer rates in Israeli BRCA1 BRCA2 mutation carrier families: is the risk increased in non-carriers? Breast Cancer Res Treat 2012;132:669-673

15. Domchek SM, Gaudet MM, Stopfer JE, et al. Breast cancer risks in individuals testing negative for a known family mutation in BRCA1 or BRCA2. Breast Cancer Res Treat 2010;119:409-414.

16. Harvey SL, Milne RL, McLachlan SA, et al. Prospective study of breast cancer risk for mutation negative women from BRCA1 or BRCA2 mutation positive families. Breast Cancer Res Treat 2011;130:1057-1061.

17. Korde LA, Mueller CM, Loud JT, et al. No evidence of excess breast cancer risk among mutation-negative women from BRCA mutationpositive families. Breast Cancer Res Treat 2011;125:169-173.

18. Nielsen HR, Petersen J, Krogh L, Nilbert M \& Skytte AB. No evidence of increased breast cancer risk for proven noncarriers from BRCA1 and BRCA2 families. Fam Cancer 2016;15:523-528.

19. Evans DG, Ingham SL, Buchan I, et al. Increased rate of phenocopies in all age groups in BRCA1/BRCA2 mutation kindred, but increased prospective breast cancer risk is confined to BRCA2 mutation carriers. Cancer Epidemiol Biomarkers Prev 2013;22:2269-2276.

20. Rowan E, Poll A \& Narod SA. A prospective study of breast cancer risk in relatives of BRCA1/BRCA2 mutation carriers. J Med Genet 2007;44:e89.

21. Vos JR, de Bock GH, Teixeira N, et al. Proven non-carriers in BRCA families have an earlier age of onset of breast cancer. Eur J Cancer 2013;49:2101-2106.

22. van der Kolk DM, de Bock GH, Leegte BK, et al. Penetrance of breast cancer, ovarian cancer and contralateral breast cancer in BRCA1 and BRCA2 families: high cancer incidence at older age. Breast Cancer Res Treat 2010;124:643-651.

23. Office for National Statistics. Cancer registration statistics, England, 2015. https://www.ons.gov.uk/peoplepopulationandcommunity/healthandsocial care/conditionsanddiseases/datasets/cancerregistrationstatisticscancerregis trationstatisticsengland. Accessed 1 March 2017.

24. Maclnnis RJ, Bickerstaffe A, Apicella C, et al. Prospective validation of the breast cancer risk prediction model BOADICEA and a batch-mode version BOADICEA Centre. Br J Cancer 2013;109:1296-1301.

25. Berliner JL \& Fay AM. Risk assessment and genetic counseling for hereditary breast and ovarian cancer: recommendations of the National Society of Genetic Counselors. J Genet Couns 2007;16:241-260.

26. Nelson HD FR, Goddard K, Mitchell Priest J, Okinaka-Hu L, Pappas M \& Zakher B. Risk assessment, genetic counseling, and genetic testing for $B R C A$ - 


\section{ORIGINAL RESEARCH ARTICLE}

related cancer: systematic review to update the U.S. Preventive Services Task Force recommendation. Evidence synthesis No. 101. Agency for Healthcare Research and Quality: Rockville (US), Publication No. 12-05164-EF-1, December 2013

27. United States Preventive Services Task Force. Final recommendation statement: BRCA-related cancer: risk assessment, genetic counseling, and genetic testing, 2013. http://www.uspreventiveservicestaskforce.org/ Page/Document/RecommendationStatementFinal/brca-related-cancer-riskassessment-genetic-counseling-and-genetic-testing. Accessed 1 March 2017.

28. Rocca W, Grossardt BR, de Andrade M, Malkasian GD \& Melton III $\sqcup$. Survival patterns after oophorectomy in premenopausal women: a population-based cohort study. Lancet Oncol 2006;7:821-828.

29. Stan DL, Shuster LT, Wick MJ, Swanson CL, Pruthi S \& Bakkum-Gamez JN. Challenging and complex decisions in the management of the BRCA mutation carrier. J Womens Health (Larchmt) 2013;22: 825-834.

30. Gahm J, Wickman M \& Brandberg Y. Bilateral prophylactic mastectomy in women with inherited risk of breast cancer: prevalence of pain and discomfort, impact on sexuality, quality of life and feelings of regret two years after surgery. Breast 2010;19:462-469.

31. Lee AJ, Cunningham AP, Kuchenbaecker KB, Mavaddat N, Easton DF \& Antoniou AC. BOADICEA breast cancer risk prediction model: updates to cancer incidences, tumour pathology and web interface. $\mathrm{Br}$ J Cancer 2014;110:535-545.
32. Tavassoli FA, Millis RR, Boecker W \& Lakhani SR. Lobular neoplasia. In Tavassoli FA, Devilee P (eds). World Health Organization Classification of Tumours. Pathology and Genetics of Tumours of the Breast and Female Genital Organs. IARC Press: Lyon, France, 2003:60-62.

33. Tavassoli FA, Hoefler $\mathrm{H}$, Rosai J, et al. Intraductal proliferative lesions. In: Tavassoli FA, Devilee P (eds). World Health Organization Classification of Tumours. Pathology and Genetics of Tumours of the Breast and Female Genital Organs. IARC Press: Lyon, France, 2003:63-73.

\section{(c) (i)}

This work is licensed under a Creative Commons Attribution 4.0 International License. The images or other third party material in this article are included in the article's Creative Commons license, unless indicated otherwise in the credit line; if the material is not included under the Creative Commons license, users will need to obtain permission from the license holder to reproduce the material. To view a copy of this license, visit http://creativecommons.org/licenses/by/4.0/

(c) The Author(s) 2018 